\title{
Ground State Solution for an Autonomous Nonlinear Schrödinger System
}

\author{
Min Liu $^{1}$ and Jiu Liu $\mathbb{D}^{2}$ \\ ${ }^{1}$ School of Mathematics and Statistics, Guizhou University, Guiyang Guizhou 550000, China \\ ${ }^{2}$ Key Laboratory of Complex Systems and Intelligent Computing, School of Mathematics and Statistics, Qiannan Normal University \\ for Nationalities, Duyun Guizhou 558000, China
}

Correspondence should be addressed to Jiu Liu; jiuliu2011@163.com

Received 18 August 2021; Accepted 7 October 2021; Published 27 October 2021

Academic Editor: Andrea Scapellato

Copyright (c) 2021 Min Liu and Jiu Liu. This is an open access article distributed under the Creative Commons Attribution License, which permits unrestricted use, distribution, and reproduction in any medium, provided the original work is properly cited.

In this paper, we study the following autonomous nonlinear Schrödinger system (discussed in the paper), where $\lambda, \mu$, and $\nu$ are positive parameters; $2^{*}=2 N /(N-2)$ is the critical Sobolev exponent; and $f$ satisfies general subcritical growth conditions. With the help of the Pohožaev manifold, a ground state solution is obtained.

\section{Introduction and Main Result}

In this paper, we consider the following autonomous nonlinear Schrödinger system:

$$
\begin{cases}-\Delta u+\mu u=\mu f(u)+\lambda v, & x \in \mathbb{R}^{N}, \\ -\Delta v+v v=|v|^{2^{*}-2} v+\lambda u, & x \in \mathbb{R}^{N}, \\ u, v \in H^{1}\left(\mathbb{R}^{N}\right), & N \geq 3\end{cases}
$$

where $\mu, v$, and $\lambda$ are positive parameters satisfying $0<\lambda<$ $\sqrt{\mu \nu} ; 2^{*}=2 N /(N-2)$ is the critical Sobolev exponent; and $f$ satisfies the following conditions:

$\left(f_{1}\right) f \in C(\mathbb{R}, \mathbb{R})$ is an odd function.

$\left(f_{2}\right) \lim _{s \longrightarrow 0^{+}}(f(s) / s)=0$.

$\left(f_{3}\right) \lim _{s \longrightarrow+\infty}\left(f(s) / s^{2^{*}-1}\right)=0$. $\left(f_{4}\right)$ There exists $\zeta>0$ such that $F(\zeta)>\left(\zeta^{2} / 2\right)$, where $F(\zeta)=\int_{0}^{\zeta} f(t) d t$

Systems of above type arise in nonlinear optics (cf. [1]). It is well known that a solution $(u, v) \in H^{1}\left(\mathbb{R}^{N}\right) \times H^{1}\left(\mathbb{R}^{N}\right)$ of system (1) is called a ground state solution if $(u, v) \neq(0$, 0 ) and its energy is minimal among the energy of all the nontrivial solutions.

The following nonlinear Schrödinger system

$$
\begin{cases}-\Delta u+\mu u=|u|^{p-1} u+\lambda v, & x \in \mathbb{R}^{N} \\ -\Delta v+v v=|v|^{q-1} v+\lambda u, & x \in \mathbb{R}^{N} \\ u, v \in H^{1}\left(\mathbb{R}^{N}\right), & \end{cases}
$$

has been studied by many authors. When $N \leq 3, \mu=v=1$, $p=q=3$, and $\lambda>0$ small enough, Ambrosetti et al. [2] proved that (2) has multibump solitons. When $N \geq 2, \mu=v$ $=1,1<p, q<2^{*}-1,0<\lambda<1$, and $|u|^{p-1} u$ and $|v|^{q-1} v$ are replaced by $(1+a(x))|u|^{p-1} u$ and $(1+b(x))|v|^{q-1} v$, Ambrosetti et al. [3] proved that system (2) has a positive ground 
state solution. When $u, v \in D^{1,2}\left(\mathbb{R}^{4}\right), \mu=V_{1}(x)$, and $v=V_{2}$ (x) satisfy the integral conditions and $|u|^{p-1} u, \lambda v,|v|^{q-1} v$ and $\lambda u$ are replaced by $\mu_{1} u^{3}, \beta u v^{2}, \mu_{2} v^{3}$, and $\beta u^{2} v$, respectively, Liu and Liu [4] proved that (2) has a positive solution. When $u, v \in H_{0}^{1}(\Omega), \Omega$ is a smooth bounded domain in $\mathbb{R}^{3}$, $p=q=3$, and $\lambda v, \lambda u$ are replaced by $-\beta u v^{2},-\beta v u^{2}$, respectively, Noris and Ramos [5] proved that (2) admits an unbounded sequence of solutions $(u, v)$ with $u>0, v>0$, and $u \neq v$ for sufficiently large $\beta>0$. When $N \geq 3,1<p<$ $2^{*}-1, q=2^{*}-1$, and $\mu, v>0,0<\lambda<\sqrt{\mu \nu}$, Chen and Zou [6] proved that (2) has a positive ground state solution under $\lambda, \mu, v$ which satisfied certain conditions. When $N \geq 3,1<$ $p<2^{*}-1, q=2^{*}-1$, and $\mu=a(x), v=b(x), \lambda=\lambda(x), \quad \mathrm{Li}$ and Tang [7] proved that (2) has a nontrivial solution.

Inspired by the above literatures, especially [6], we investigate the existence of ground state solution of system (1). When $\mu f(u)=|u|^{p-1} u$ with $1<p<2^{*}-1$, by using the Nehari manifold, Chen and Zou [6] obtained the existence of ground state solution of system (1). But in our paper, without the assumption of the monotonicity of $u \mapsto(f(u))$ $/ u$, we have to adopt a new method to replace the Nehari manifold.

The following single Schrödinger equation

$$
-\Delta u+u=f(u), \quad u \in H^{1}\left(\mathbb{R}^{N}\right), N \geq 3
$$

has been widely studied by many researchers, and relevant results can been referred to [8-10] and the references therein. By [9], we know that if $f$ satisfies $\left(f_{1}\right)-\left(f_{4}\right)$; then, equation (3) has a ground state solution. Define

$$
a=\inf _{u \in \Gamma}\left[\frac{1}{2} \int_{\mathbb{R}^{N}}\left(|\nabla u|^{2}+u^{2}\right) d x-\int_{\mathbb{R}^{N}} F(u) d x\right],
$$

where $\quad \Gamma=\left\{u \in H^{1}\left(\mathbb{R}^{N}\right): u\right.$ is a nontrivial solution of equation (3) $\}$ and define

$$
S=\inf _{u \in D^{1,2}\left(\mathbb{R}^{N}\right) \backslash\{0\}} \frac{\int_{\mathbb{R}^{N}}|\nabla u|^{2} d x}{\left(\int_{\mathbb{R}^{N}}|u|^{2^{*}} d x\right)^{2 / 2^{*}}},
$$

where $S$ is the optimal constant of the Sobolev embedding $D^{1,2}\left(\mathbb{R}^{N}\right) \longrightarrow L^{2^{*}}\left(\mathbb{R}^{N}\right)$.

The main result of this paper is the following.

Theorem 1. Assume that $\mu, v$, and $\lambda$ are positive parameters satisfying $\mu>S^{-N /(N-2)}(a N)^{2 /(N-2)}$ and $0<\lambda<\sqrt{\mu \nu}$. Suppose that $f$ satisfies $\left(f_{1}\right)-\left(f_{4}\right)$. Then, system (1) has a ground state solution.

Remark 2. There are some examples of functions that satisfy the assumptions $\left(f_{1}\right)-\left(f_{4}\right)$, for example, $f(s)=|s|^{p-2} s$ with 2 $<p<2^{*}$ and $f(s)=|s|^{p-2} s /\left(1+s^{2}\right)$ with $4<p<2^{*}+2$.

Remark 3. It is obvious that system (1) has no semitrivial solutions. Indeed, if $(u, 0)$ is a solution of system (1), then $u=0$ and if $(0, v)$ is a solution of system (1), then $v=0$.
Remark 4. There are some recent studies on the ground state solutions for other types of Schrödinger equations or systems, for example, [6, 11]. Moreover, in the bounded domain, the existence and the regularity of solutions to differential problems have been widely investigated by using tools of harmonic and real analysis and variational methods, for example, [12-14].

\section{Preliminaries}

In order to make a precise explanation of the results in this paper, we will give some notations.

$C, C_{i}$ denote various positive constants.

$L^{p}\left(\mathbb{R}^{N}\right)$ is the usual Lebesgue space endowed with the norm

$$
|u|_{p}=\left(\int_{\mathbb{R}^{N}}|u|^{p} d x\right)^{1 / p}
$$

$$
D^{1,2}\left(\mathbb{R}^{N}\right)=\left\{u \in L^{2^{*}}\left(\mathbb{R}^{N}\right) \mid\left(\partial u / \partial x_{i}\right) \in L^{2}\left(\mathbb{R}^{N}\right), i=1,2,\right.
$$

$\cdots, N\}$ endowed with the norm

$$
\|u\|_{D^{1,2}}=\left(\int_{\mathbb{R}^{N}}|\nabla u|^{2} d x\right)^{1 / 2}
$$

$$
H^{1}\left(\mathbb{R}^{N}\right)=\left\{u \in L^{2}\left(\mathbb{R}^{N}\right) \mid\left(\partial u / \partial x_{i}\right) \in L^{2}\left(\mathbb{R}^{N}\right), i=1,2, \cdots,\right.
$$

$N\}$ endowed with the norm

$$
\|u\|=\left(\int_{\mathbb{R}^{N}}\left(|\nabla u|^{2}+u^{2}\right) d x\right)^{1 / 2}
$$

For any $(u, v) \in H:=H^{1}\left(\mathbb{R}^{N}\right) \times H^{1}\left(\mathbb{R}^{N}\right)$, we set

$$
\|u, v\|_{H}=\left(\int_{\mathbb{R}^{N}}\left(|\nabla u|^{2}+\mu u^{2}+|\nabla v|^{2}+v v^{2}\right) d x\right)^{1 / 2} .
$$

For any $u \in H^{1}\left(\mathbb{R}^{N}\right)$, we denote $u_{t}=u(\cdot / t)$ for all $t>0$.

The weak solutions of (1) correspond to critical points of the functional

$I(u, v)=\frac{1}{2}\|u, v\|_{H}^{2}-\mu \int_{\mathbb{R}^{N}} F(u) d x-\frac{1}{2^{*}} \int_{\mathbb{R}^{N}}|v|^{2^{*}} d x-\lambda \int_{\mathbb{R}^{N}} u v d x$.

Obviously, $I \in C^{1}(H, \mathbb{R})$ and for all $(u, v) \in H$ and $(\varphi, \psi)$ $\in H$, we have

$$
\begin{aligned}
\left\langle I^{\prime}(u, v),(\varphi, \psi)\right\rangle= & \int_{\mathbb{R}^{N}}(\nabla u \cdot \nabla \varphi+\mu u \varphi+\nabla v \cdot \nabla \psi+\nu v \psi) d x \\
& -\mu \int_{\mathbb{R}^{N}} f(u) \varphi d x-\int_{\mathbb{R}^{N}}|v|^{2^{*}-2} v \psi d x \\
& -\lambda \int_{\mathbb{R}^{N}}(\varphi v+u \psi) d x .
\end{aligned}
$$

Similar to $[15,16]$, in order to obtain a ground state 
solution, we define the Pohožaev manifold

$$
\mathscr{P}=\{(u, v) \in H \backslash\{(0,0)\}: J(u, v)=0\}
$$

and consider the constraint minimization problem

$$
m=\inf _{(u, v) \in \mathscr{P}} I(u, v)
$$

where $J: H \longrightarrow \mathbb{R}$ is defined as

$$
\begin{aligned}
J(u, v)= & \frac{N-2}{2} \int_{\mathbb{R}^{N}}\left(|\nabla u|^{2}+|\nabla v|^{2}\right) d x+\frac{N}{2} \int_{\mathbb{R}^{N}}\left(\mu u^{2}+v v^{2}\right) d x \\
& -\mu N \int_{\mathbb{R}^{N}} F(u) d x-\frac{N}{2^{*}} \int_{\mathbb{R}^{N}}|v|^{2^{*}} d x-\lambda N \int_{\mathbb{R}^{N}} u v d x .
\end{aligned}
$$
(1):

We also require the following subcritical system of system

$$
\begin{cases}-\Delta u+\mu u=\mu f(u)+\lambda v, & x \in \mathbb{R}^{N}, \\ -\Delta v+v v=|v|^{q-2} v+\lambda u, & x \in \mathbb{R}^{N}, \\ u, v \in H^{1}\left(\mathbb{R}^{N}\right), & N \geq 3,\end{cases}
$$

where $2<q<2^{*}, \mu, v$, and $\lambda$ are positive parameters satisfying $0<\lambda<\sqrt{\mu \nu}$ and $f$ satisfies $\left(f_{1}\right)-\left(f_{4}\right)$. The energy functional of system (15) is

$I_{q}(u, v)=\frac{1}{2}\|u, v\|_{H}^{2}-\mu \int_{\mathbb{R}^{N}} F(u) d x-\frac{1}{q} \int_{\mathbb{R}^{N}}|v|^{q} d x-\lambda \int_{\mathbb{R}^{N}} u v d x$.

Define

$$
\begin{aligned}
\mathscr{P}_{q} & =\left\{(u, v) \in H \backslash\{(0,0)\}: J_{q}(u, v)=0\right\} \text { and } m_{q} \\
& =\inf _{(u, v) \in \mathscr{P}_{q}} I_{q}(u, v),
\end{aligned}
$$

where

$$
\begin{aligned}
J_{q}(u, v)= & \frac{N-2}{2} \int_{\mathbb{R}^{N}}\left(|\nabla u|^{2}+|\nabla v|^{2}\right) d x+\frac{N}{2} \int_{\mathbb{R}^{N}}\left(\mu u^{2}+v v^{2}\right) d x \\
& -\mu N \int_{\mathbb{R}^{N}} F(u) d x-\frac{N}{q} \int_{\mathbb{R}^{N}}|v|^{q} d x-\lambda N \int_{\mathbb{R}^{N}} u v d x .
\end{aligned}
$$

\section{Proof of Theorem 1}

The following two lemmas will be used in proof.

Lemma 5 (compactness lemma of Strauss, see [9, 10]). Let $P, Q: \mathbb{R} \longrightarrow \mathbb{R}$ be two continuous functions satisfying

$$
\frac{P(s)}{Q(s)} \longrightarrow 0 \quad \text { as }|s| \longrightarrow+\infty
$$

Let $u_{n}$ be a sequence of measurable functions: $\mathbb{R}^{N} \longrightarrow \mathbb{R}$ such that

$$
\sup _{n} \int_{\mathbb{R}^{N}}\left|Q\left(u_{n}(x)\right)\right| d x<+\infty
$$

and $P\left(u_{n}(x)\right) \longrightarrow v(x)$ a.e. in $\mathbb{R}^{N}$, as $n \longrightarrow \infty$. Then, for any bounded Borel set $B$, one has

$$
\int_{B}\left|P\left(u_{n}(x)\right)-v(x)\right| d x \longrightarrow 0 \text { as } n \longrightarrow+\infty
$$

If one further assumes that

$$
\frac{P(s)}{Q(s)} \longrightarrow 0 \quad \text { as }|s| \longrightarrow 0
$$

and $u_{n}(x) \longrightarrow 0$ as $|x| \longrightarrow+\infty$, uniformly with respect to $n$, then $P\left(u_{n}\right)$ converges to $v$ in $L^{1}\left(\mathbb{R}^{N}\right)$ as $n \longrightarrow+\infty$.

Lemma 6 (Strauss inequality, see [17]). If $N \geq 2$, there exists $C_{N}>0$ such that, for every $u(x)=u(|x|) \in H^{1}\left(\mathbb{R}^{N}\right)$,

$$
|u(x)| \leq C_{N}|u|_{2}^{1 / 2}|\nabla u|_{2}^{1 / 2}|x|^{(1-N) / 2}
$$

a.e. on $\mathbb{R}^{N}$.

Before proving Theorem 1, we need to prove a series of lemmas.

Lemma 7. Suppose that $\left(f_{1}\right)$ - $\left(f_{4}\right)$ hold. Then, the Pohožaev manifold $\mathscr{P}$ is not empty.

Proof. From [17], we know that for any $\varepsilon>0$,

$$
u_{\varepsilon}=\frac{[N(N-2)]^{(N-2) / 4} \varepsilon^{(N-2) / 2}}{\left(\varepsilon+|x|^{2}\right)^{(N-2) / 4}}
$$

is a positive solution of the following equation:

$$
-\Delta u=|u|^{2^{*}-2} u, \quad x \in \mathbb{R}^{N}, N \geq 3 .
$$

Define a cut-off function $\phi \in C_{0}^{\infty}\left(\mathbb{R}^{N},[0,1]\right)$ as

$$
\phi= \begin{cases}1, & x \in B_{\rho}, \\ 0, & x \in \mathbb{R}^{N} \backslash B_{2 \rho},\end{cases}
$$

where $\mathrm{\varrho}>0$ and $B_{\mathrm{Q}}=\left\{x \in \mathbb{R}^{N},|x|<\mathrm{Q}\right\}$. Let $W_{\varepsilon}=\phi u_{\varepsilon}$ and 
define $V_{\varepsilon}=W_{\varepsilon} /\left(\int_{\mathbb{R}^{N}}\left|W_{\varepsilon}\right|^{2^{*}} d x\right)^{1 / 2^{*}}$. By [16], we have

$$
\begin{gathered}
\left(\int_{\mathbb{R}^{N}}\left|V_{\varepsilon}\right|^{2^{*}} d x\right)^{1 / 2^{*}}=1, \\
\int_{\mathbb{R}^{N}}\left|V_{\varepsilon}\right|^{2} d x= \begin{cases}o\left(\varepsilon^{1 / 2}\right), & N=3, \\
o(\varepsilon|\ln \varepsilon|), & N=4, \\
o(\varepsilon), & N=5 .\end{cases}
\end{gathered}
$$

Take $\varepsilon>0$ small enough such that $\int_{\mathbb{R}^{N}}\left(\left(1 / 2^{*}\right)\left|V_{\varepsilon}\right|^{2^{*}}-\right.$ $\left.(v / 2) V_{\varepsilon}^{2}\right) d x>0$. Let $U \in H^{1}\left(\mathbb{R}^{N}\right)$ be a positive ground state solution of equation (3). Then, we have the following Pohožaev equality:

$$
\frac{N-2}{2} \int_{\mathbb{R}^{N}}|\nabla U|^{2} d x+\frac{N}{2} \int_{\mathbb{R}^{N}} U^{2} d x=N \int_{\mathbb{R}^{N}} F(U) d x .
$$

Then, $\int_{\mathbb{R}^{N}}\left(F(U)-(1 / 2) U^{2}\right) d x>0$. Thus, we have

$$
\begin{aligned}
\tau(t):= & I\left(U_{t},\left(V_{\varepsilon}\right)_{t}\right)=\frac{t^{N-2}}{2} \int_{\mathbb{R}^{N}}\left(|\nabla U|^{2}+\left|\nabla V_{\varepsilon}\right|^{2}\right) d x \\
& -\mu t^{N} \int_{\mathbb{R}^{N}}\left(F(U)-\frac{1}{2} U^{2}\right) d x \\
& -t^{N} \int_{\mathbb{R}^{N}}\left(\frac{1}{2^{*}}\left|V_{\varepsilon}\right|^{2^{*}}-\frac{v}{2} V_{\varepsilon}^{2}\right) d x-\lambda t^{N} \int_{\mathbb{R}^{N}} U V_{\varepsilon} d x .
\end{aligned}
$$

Define $s=t^{N}$; we have

$$
\begin{aligned}
\eta(s):= & \tau\left(s^{1 / N}\right)=\frac{s^{(N-2) / N}}{2} \int_{\mathbb{R}^{N}}\left(|\nabla U|^{2}+\left|\nabla V_{\varepsilon}\right|^{2}\right) d x \\
& -\mu s \int_{\mathbb{R}^{N}}\left(F(U)-\frac{1}{2} U^{2}\right) d x \\
& -s \int_{\mathbb{R}^{N}}\left(\frac{1}{2^{*}}\left|V_{\varepsilon}\right|^{2^{*}}-\frac{v}{2} V_{\varepsilon}^{2}\right) d x-\lambda s \int_{\mathbb{R}^{N}} U V_{\varepsilon} d x .
\end{aligned}
$$

We can easily know that $\eta(s)>0$ for $s$ small enough and $\eta(s)<0$ for large $s$. Since $\left(d^{2} \eta(s)\right) / d s^{2}<0, \eta(s)$ is a concave function. Then, there exists a unique $s_{0}>0$ such that $\eta^{\prime}\left(s_{0}\right)=0$. Hence, there exists a unique $t_{0}=s_{0}^{1 / N}>0$ such that $\tau^{\prime}\left(t_{0}\right)=0$. Then, we have $t_{0} \tau^{\prime}\left(t_{0}\right)=J\left(U\left(x / t_{0}\right)\right.$, $\left.V_{\varepsilon}\left(x / t_{0}\right)\right)=0$. Then, $\left(U_{t_{0}},\left(V_{\varepsilon}\right)_{t_{0}}\right) \in \mathscr{P}$.

Lemma 8. Suppose that $\left(f_{1}\right)-\left(f_{4}\right)$ hold. Then, $m=\inf _{(u, v) \in \mathscr{P}} I$ $(u, v)>0$.

Proof. Since $0<\lambda<\sqrt{\mu \nu}$, there exists $0<\theta<1$ such that 0 $<\lambda<\sqrt{\mu(1-\theta) v}$. For any $(u, v) \in \mathscr{P}$, we have $J(u, v)=0$.
By using Young's inequality, we have

$$
\begin{aligned}
& \frac{N-2}{2} \int_{\mathbb{R}^{N}}\left(|\nabla u|^{2}+|\nabla v|^{2}\right) d x+\frac{N}{2} \int_{\mathbb{R}^{N}}\left(\mu u^{2}+v v^{2}\right) d x \\
& =\mu N \int_{\mathbb{R}^{N}} F(u) d x+\frac{N}{2^{*}} \int_{\mathbb{R}^{N}}|v|^{2^{*}} d x+\lambda N \int_{\mathbb{R}^{N}} u v d x \\
& \leq \mu N \frac{\theta}{2} \int_{\mathbb{R}^{N}} u^{2} d x+N C \int_{\mathbb{R}^{N}}|u|^{2^{*}} d x+\frac{N}{2^{*}} \int_{\mathbb{R}^{N}}|v|^{2^{*}} d x \\
& \quad+\lambda N \int_{\mathbb{R}^{N}} u v d x \leq \mu N \frac{\theta}{2} \int_{\mathbb{R}^{N}} u^{2} d x+N C \int_{\mathbb{R}^{N}}|u|^{2^{*}} d x \\
& \quad+\frac{N}{2^{*}} \int_{\mathbb{R}^{N}}|v|^{2^{*}} d x+N \frac{\mu(1-\theta)}{2} \int_{\mathbb{R}^{N}} u^{2} d x+\frac{N v}{2} \int_{\mathbb{R}^{N}} v^{2} d x .
\end{aligned}
$$

Therefore, we have

$\frac{N-2}{2} \int_{\mathbb{R}^{N}}\left(|\nabla u|^{2}+|\nabla v|^{2}\right) d x \leq N C \int_{\mathbb{R}^{N}}|u|^{2^{*}} d x+\frac{N}{2^{*}} \int_{\mathbb{R}^{N}}|v|^{2^{*}} d x$.

By using Sobolev's inequality, we have

$$
\begin{aligned}
& \frac{N-2}{2} \int_{\mathbb{R}^{N}}\left(|\nabla u|^{2}+|\nabla v|^{2}\right) d x \\
& \quad \leq C_{1}\left[\left(\int_{\mathbb{R}^{N}}|\nabla u|^{2} d x\right)^{2^{*} / 2}+\left(\int_{\mathbb{R}^{N}}|\nabla v|^{2} d x\right)^{2^{*} / 2}\right] \\
& \quad \leq C_{2}\left[\int_{\mathbb{R}^{N}}\left(|\nabla u|^{2}+|\nabla v|^{2}\right) d x\right]^{2^{*} / 2},
\end{aligned}
$$

which implies $\int_{\mathbb{R}^{N}}\left(|\nabla u|^{2}+|\nabla v|^{2}\right) d x \geq\left((N-2) / 2 C_{2}\right)^{(N-2) / 2}$ $>0$. Therefore, we conclude that for any $(u, v) \in \mathscr{P}$, we have

$$
\begin{aligned}
I(u, v)= & I(u, v)-\frac{1}{N} J(u, v)=\left(\frac{1}{2}-\frac{1}{2^{*}}\right) \int_{\mathbb{R}^{N}}|\nabla u|^{2} d x \\
& +\left(\frac{1}{2}-\frac{1}{2^{*}}\right) \int_{\mathbb{R}^{N}}|\nabla v|^{2} d x \geq \frac{1}{N}\left(\frac{N-2}{2 C_{2}}\right)^{(N-2) / 2}
\end{aligned}
$$

Therefore, we have $m>0$.

Lemma 9. Suppose that $\left(f_{1}\right)-\left(f_{4}\right)$ hold. Then, $m<(1 / N) S^{N / 2}$.

Proof. Let $U \in H^{1}\left(\mathbb{R}^{N}\right)$ be a positive ground state solution of equation (3). Then, (28) holds and

$$
\begin{aligned}
a= & \frac{1}{2} \int_{\mathbb{R}^{N}}\left(|\nabla U|^{2}+U^{2}\right) d x-\int_{\mathbb{R}^{N}} F(U) d x \\
= & \frac{1}{2} \int_{\mathbb{R}^{N}}\left(|\nabla U|^{2}+U^{2}\right) d x-\int_{\mathbb{R}^{N}} F(U) d x \\
& -\frac{1}{N}\left(\frac{N-2}{2} \int_{\mathbb{R}^{N}}|\nabla U|^{2} d x+\frac{N}{2} \int_{\mathbb{R}^{N}} U^{2} d x-N \int_{\mathbb{R}^{N}} F(U) d x\right) \\
= & \int_{\mathbb{R}^{N}}|\nabla U|^{2} d x .
\end{aligned}
$$


Moreover, we have also $U(\sqrt{\mu} x)$ which is a solution of equation

$$
-\Delta u+\mu u=\mu f(u), \quad u \in H^{1}\left(\mathbb{R}^{N}\right), N \geq 3 .
$$

Then, $(U(\sqrt{\mu} x), 0) \in \mathscr{P}$. Since $\mu>S^{-N /(N-2)}(\mathrm{aN})^{2 /(N-2)}$, we have

$$
\begin{aligned}
m & \leq I(U(\sqrt{\mu} x), 0)=I(U(\sqrt{\mu} x), 0)-\frac{1}{N} J(U(\sqrt{\mu} x), 0) \\
& =\frac{1}{N} \int_{\mathbb{R}^{N}}|\nabla U(\sqrt{\mu} x)|^{2} d x=a \mu^{(2-N) / 2}<\frac{1}{N} S^{N / 2} .
\end{aligned}
$$

Lemma 10. Suppose that $\left(f_{1}\right)-\left(f_{4}\right)$ hold. For any $\left(u_{n}, v_{n}\right) \subset \mathscr{P}$, if $I\left(u_{n}, v_{n}\right) \leq C$, then $\left(u_{n}, v_{n}\right)$ is bounded in $H$.

Proof. Since $I\left(u_{n}, v_{n}\right) \leq C$, we have

$$
\begin{aligned}
C & \geq I\left(u_{n}, v_{n}\right)=I\left(u_{n}, v_{n}\right)-\frac{1}{N} J\left(u_{n}, v_{n}\right) \\
& =\frac{1}{N} \int_{\mathbb{R}^{N}}\left(\left|\nabla u_{n}\right|^{2}+\left|\nabla v_{n}\right|^{2}\right) d x .
\end{aligned}
$$

Because $0<\lambda<\sqrt{\mu \nu}$, there exists $0<\theta<1 / 2$ and $\alpha>0$ such that $0<\lambda<\sqrt{\mu(1-2 \theta)(\nu-\alpha)}$. Therefore, we have

$$
\begin{aligned}
\frac{N-2}{2} \int_{\mathbb{R}^{N}}\left(\left|\nabla u_{n}\right|^{2}+\left|\nabla v_{n}\right|^{2}\right) d x+\frac{N}{2} \int_{\mathbb{R}^{N}}\left(\mu u_{n}^{2}+v v_{n}^{2}\right) d x \\
=\mu N \int_{\mathbb{R}^{N}} F\left(u_{n}\right) d x+\frac{N}{2^{*}} \int_{\mathbb{R}^{N}}\left|v_{n}\right|^{2^{*}} d x+\lambda N \int_{\mathbb{R}^{N}} u_{n} v_{n} d x \\
\leq \mu N \frac{\theta}{2} \int_{\mathbb{R}^{N}}\left|u_{n}\right|^{2} d x+N C \int_{\mathbb{R}^{N}}\left|u_{n}\right|^{2^{*}} d x+\frac{N}{2^{*}} \int_{\mathbb{R}^{N}}\left|v_{n}\right|^{2^{*}} d x \\
\quad+\lambda N \int_{\mathbb{R}^{N}} u_{n} v_{n} d x \leq \mu N \frac{\theta}{2} \int_{\mathbb{R}^{N}}\left|u_{n}\right|^{2} d x \\
\quad+N C \int_{\mathbb{R}^{N}}\left|u_{n}\right|^{2^{*}} d x+\frac{N}{2^{*}} \int_{\mathbb{R}^{N}}\left|v_{n}\right|^{2^{*}} d x \\
\quad+N \frac{\mu(1-2 \theta)}{2} \int_{\mathbb{R}^{N}} u_{n}^{2} d x+\frac{N(v-\alpha)}{2} \int_{\mathbb{R}^{N}} v_{n}^{2} d x .
\end{aligned}
$$

Then, we have

$$
\begin{aligned}
\frac{N \mu \theta}{2} \int_{\mathbb{R}^{N}}\left|u_{n}\right|^{2} d x+\frac{N \alpha}{2} \int_{\mathbb{R}^{N}}\left|v_{n}\right|^{2} d x \leq C N \int_{\mathbb{R}^{N}}\left|u_{n}\right|^{2^{*}} d x \\
+\frac{N}{2^{*}} \int_{\mathbb{R}^{N}}\left|v_{n}\right|^{2^{*}} d x \leq C_{3}\left[\int_{\mathbb{R}^{N}}\left(|\nabla u|^{2}+|\nabla v|^{2}\right) d x\right]^{2^{*} / 2} \leq C_{4} .
\end{aligned}
$$

Hence, $\left(u_{n}, v_{n}\right)$ is bounded in $H$.

Lemma 11. Suppose that $\left(f_{1}\right)-\left(f_{4}\right)$ hold. Then, $\lim _{q \longrightarrow 2^{*-}} \sup m_{q}$ $\leq m$
Proof. For any $\varepsilon \in(0,1 / 2)$, there exists $(u, v) \in \mathscr{P}$ such that $I(u, v)<m+\varepsilon$. Since $J(u, v)=0$, for any $t>0$, we have

$$
\begin{aligned}
I\left(u_{t}, v_{t}\right) & =I\left(u_{t}, v_{t}\right)-\frac{t^{N}}{N} J(u, v) \\
& =\left(\frac{t^{N-2}}{2}-\frac{N-2}{2 N} t^{N}\right) \int_{\mathbb{R}^{N}}\left(|\nabla u|^{2}+|\nabla v|^{2}\right) d x .
\end{aligned}
$$

Define $h(t)=t^{N-2} / 2-((N-2) / 2 N) t^{N}$. Through simple calculations, we have $h^{\prime}(t)=(N-2) / 2\left(t^{N-3}-t^{N-1}\right)$. We can easily see that $h$ is increasing for $t \in(0,1)$ and $h$ is decreasing for $t>1$. Then, we have $\max _{t>0} I\left(u_{t}, v_{t}\right)=I(u, v)$ and $I\left(u_{t}, v_{t}\right)<I(u, v)$ for any $t \neq 1$. By calculation, we have $I\left(u_{t}, v_{t}\right)<0$ for $t>\sqrt{N /(N-2)}$. Take large $T$ such that

$$
\begin{aligned}
I\left(u_{T}, v_{T}\right)= & \frac{T^{N-2}}{2} \int_{\mathbb{R}^{N}}\left(|\nabla u|^{2}+|\nabla v|^{2}\right) d x \\
& +\frac{T^{N}}{2} \int_{\mathbb{R}^{N}}\left(\mu u^{2}+v v^{2}\right) d x-\mu T^{N} \int_{\mathbb{R}^{N}} F(u) d x \\
& -\frac{T^{N}}{2^{*}} \int_{\mathbb{R}^{N}}|v|^{2^{*}} d x-\lambda T^{N} \int_{\mathbb{R}^{N}} u v d x \leq-1 .
\end{aligned}
$$

Then, there exists $\sigma \in\left(0,2^{*}\right)$ such that

$$
\left|I_{q}\left(u_{t}, v_{t}\right)-I\left(u_{t}, v_{t}\right)\right|=\left.\left|\frac{t^{N}}{2^{*}} \int_{\mathbb{R}^{N}}\right| v\right|^{2^{*}} d x-\frac{t^{N}}{q} \int_{\mathbb{R}^{N}}|v|^{q} d x \mid<\varepsilon,
$$

for all $2^{*}-\sigma<q<2^{*}$ and $0 \leq t \leq T$. Then, we have $I_{q}\left(u_{T}\right.$, $\left.v_{T}\right) \leq-(1 / 2)$ for all $2^{*}-\sigma<q<2^{*}$. Since

$$
\begin{aligned}
I_{q}\left(u_{t}, v_{t}\right)= & \frac{t^{N-2}}{2} \int_{\mathbb{R}^{N}}\left(|\nabla u|^{2}+|\nabla v|^{2}\right) d x \\
& +\frac{t^{N}}{2} \int_{\mathbb{R}^{N}}\left(\mu u^{2}+v v^{2}\right) d x-\mu t^{N} \int_{\mathbb{R}^{N}} F(u) d x \\
& -\frac{t^{N}}{q} \int_{\mathbb{R}^{N}}|v|^{q} d x-\lambda t^{N} \int_{\mathbb{R}^{N}} u v d x,
\end{aligned}
$$

$I_{q}\left(u_{t}, v_{t}\right)>0$ for $t$ small enough. Then, there exists $t_{q} \in(0, T)$ such that $\left.(d / d t) I_{q}\left(u_{t}, v_{t}\right)\right|_{t=t_{q}}=0$. So, $\left(u_{t_{q}}, v_{t_{q}}\right) \in \mathscr{P}_{q}$. Hence, we have

$$
m_{q} \leq I_{q}\left(u_{t_{q}}, v_{t_{q}}\right) \leq I\left(u_{t_{q}}, v_{t_{q}}\right)+\varepsilon \leq I(u, v)+\varepsilon<m+2 \varepsilon,
$$

for all $2^{*}-\sigma<q<2^{*}$.

From $[18,19]$, we know that system (15) has a positive and radial ground state solution. Then, for any $q_{n} \in\left(2,2^{*}\right)$ and $q_{n} \longrightarrow 2^{*-}$, there exists a positive and radial sequence $\left\{\left(u_{n}, v_{n}\right)\right\} \subset H$ such that 


$$
\begin{gathered}
I_{q_{n}}\left(u_{n}, v_{n}\right)=m_{q_{n}}, \\
I_{q_{n}}^{\prime}\left(u_{n}, v_{n}\right)=0, \\
J_{q_{n}}\left(u_{n}, v_{n}\right)=0 .
\end{gathered}
$$

By Lemmas 10 and 11 , we know that $\left\{\left(u_{n}, v_{n}\right)\right\}$ is bounded in $H$.

Lemma 12. Suppose that $\left(f_{1}\right)-\left(f_{4}\right)$ and (46) hold. Then, $\liminf _{n \rightarrow \infty} m_{q_{n}}>0$.

Proof. Similar to the proof of Lemma 8, we have

$$
\begin{gathered}
\frac{N-2}{2} \int_{\mathbb{R}^{N}}\left(\left|\nabla u_{n}\right|^{2}+\left|\nabla v_{n}\right|^{2}\right) d x \leq N C \int_{\mathbb{R}^{N}}\left|u_{n}\right|^{2^{*}} d x \\
+\frac{N}{q_{n}} \int_{\mathbb{R}^{N}}\left|v_{n}\right|^{q_{n}} d x .
\end{gathered}
$$

Using Young's inequality implies

$$
\begin{aligned}
& \frac{N}{q_{n}} \int_{\mathbb{R}^{N}}\left|v_{n}\right|^{q_{n}} d x=\frac{N}{q_{n}} \int_{\mathbb{R}^{N}}\left|v_{n}\right|^{\left(2\left(2^{*}-q_{n}\right)\right) /\left(2^{*}-2\right)}\left|v_{n}\right|^{\left(2^{*}\left(q_{n}-2\right)\right) /\left(2^{*}-2\right)} d x \\
& \quad \leq \frac{N}{q_{n}} \frac{2^{*}-q_{n}}{2^{*}-2} \int_{\mathbb{R}^{N}}\left|v_{n}\right|^{2} d x+\frac{N}{q_{n}} \frac{q_{n}-2}{2^{*}-2} \int_{\mathbb{R}^{N}}\left|v_{n}\right|^{2^{*}} d x \\
& \quad=\frac{N}{2^{*}} \int_{\mathbb{R}^{N}}\left|v_{n}\right|^{2^{*}} d x+o(1) .
\end{aligned}
$$

Then,

$$
\begin{aligned}
& \frac{N-2}{2} \int_{\mathbb{R}^{N}}\left(\left|\nabla u_{n}\right|^{2}+\left|\nabla v_{n}\right|^{2}\right) d x \leq N C \int_{\mathbb{R}^{N}}\left|u_{n}\right|^{2^{*}} d x \\
& \quad+\frac{N}{2^{*}} \int_{\mathbb{R}^{N}}\left|v_{n}\right|^{2^{*}} d x+o(1) \leq C_{5}\left[\int_{\mathbb{R}^{N}}\left(\left|\nabla u_{n}\right|^{2}+\left|\nabla v_{n}\right|^{2}\right) d x\right]^{2^{*} / 2}+o(1) .
\end{aligned}
$$

So there exists $\omega>0$ such that up to a subsequence, $\int_{\mathbb{R}^{N}}\left(\left|\nabla u_{n}\right|^{2}+\left|\nabla v_{n}\right|^{2}\right) d x+o(1) \geq \omega$. On the other hand,

$$
\begin{aligned}
m_{q_{n}} & =I_{q_{n}}\left(u_{n}, v_{n}\right)=I_{q_{n}}\left(u_{n}, v_{n}\right)-\frac{1}{N} J_{q_{n}}\left(u_{n}, v_{n}\right) \\
& =\frac{1}{N} \int_{\mathbb{R}^{N}}\left(\left|\nabla u_{n}\right|^{2}+\left|\nabla v_{n}\right|^{2}\right) d x .
\end{aligned}
$$

Then, $\liminf _{n \rightarrow \infty} m_{q_{n}}>0$.

Proof of Theorem 1. Because (46) holds, there exists $(u, v)$ $\in H$ such that $\mathrm{u}_{n} u, v_{n} v$ in $H^{1}\left(\mathbb{R}^{N}\right), u_{n} \longrightarrow u, v_{n} \longrightarrow v$ in $L^{p}$ $\left(\mathbb{R}^{N}\right), 2<p<2^{*}$, and $u_{n}(x) \longrightarrow u(x), v_{n}(x) \longrightarrow v(x)$ a.e. in $\mathbb{R}^{N}$. For any $(\varphi, \psi) \in H$, we have

$$
0=\left\langle I_{q_{n}}^{\prime}\left(u_{n}, v_{n}\right),(\varphi, \psi)\right\rangle \longrightarrow\left\langle I^{\prime}(u, v),(\varphi, \psi)\right\rangle,
$$

i.e., $(u, v)$ is a solution of system (1). Suppose that $u=0$. Set $P(s)=f(s) s$ and $Q(s)=|s|^{2}+|s|^{2^{*}}$. Through Lemma 5 and Lemma 6 , we have $\int_{\mathbb{R}^{N}} P\left(u_{n}\right) d x \longrightarrow 0$ as $n \longrightarrow+\infty$. Since $<$ $\left.I_{q_{n}}^{\prime}\left(u_{n}, v_{n}\right),\left(u_{n}, v_{n}\right)\right\rangle=0$, by using Young's inequality, we have

$$
\begin{aligned}
\left\|u_{n}, v_{n}\right\|_{H}= & \mu \int_{\mathbb{R}^{N}} f\left(u_{n}\right) u_{n} d x+\int_{\mathbb{R}^{N}}\left|v_{n}\right|^{q_{n}} d x+2 \lambda \int_{\mathbb{R}^{N}} u_{n} v_{n} d x \\
\leq & \mu \int_{\mathbb{R}^{N}} P\left(u_{n}\right) d x+\int_{\mathbb{R}^{N}}\left|v_{n}\right|^{\left(2\left(2^{*}-q_{n}\right)\right) /\left(2^{*}-2\right)}\left|v_{n}\right|^{\left(2^{*}\left(q_{n}-2\right)\right) /\left(2^{*}-2\right)} d x \\
& +\int_{\mathbb{R}^{N}}\left(\mu u_{n}^{2}+v v_{n}^{2}\right) d x \leq \mu \int_{\mathbb{R}^{N}} P\left(u_{n}\right) d x+\frac{2^{*}-q_{n}}{2^{*}-2} \int_{\mathbb{R}^{N}}\left|v_{n}\right|^{2} d x \\
& +\frac{q_{n}-2}{2^{*}-2} \int_{\mathbb{R}^{N}}\left|v_{n}\right|^{2^{*}} d x+\int_{\mathbb{R}^{N}}\left(\mu u_{n}^{2}+v v_{n}^{2}\right) d x=\int_{\mathbb{R}^{N}}\left|v_{n}\right|^{2^{*}} d x \\
& +\int_{\mathbb{R}^{N}}\left(\mu u_{n}^{2}+v v_{n}^{2}\right) d x+o(1) .
\end{aligned}
$$

One has

$$
\begin{aligned}
\int_{\mathbb{R}^{N}}\left|\nabla v_{n}\right|^{2} d x & \leq \int_{\mathbb{R}^{N}}\left(\left|\nabla u_{n}\right|^{2}+\left|\nabla v_{n}\right|^{2}\right) d x \leq \int_{\mathbb{R}^{N}}\left|v_{n}\right|^{2^{*}} d x+o(1) \\
& \leq\left(\frac{\int_{\mathbb{R}^{N}}\left|\nabla v_{n}\right|^{2} d x}{S}\right)^{2^{*} / 2}+o(1)
\end{aligned}
$$

So we have (i) $\int_{\mathbb{R}^{N}}\left|\nabla v_{n}\right|^{2} d x \longrightarrow 0$ or (ii) $\lim \sup \int_{\mathbb{R}^{N}}$ $\left|\nabla v_{n}\right|^{2} d x \geq S^{N / 2}$. If (i) holds, then we have

$$
\begin{aligned}
m_{q_{n}} & =I_{q_{n}}\left(u_{n}, v_{n}\right)-\frac{1}{N} J_{q_{n}}\left(u_{n}, v_{n}\right) \\
& =\frac{1}{N} \int_{\mathbb{R}^{N}}\left(\left|\nabla u_{n}\right|^{2}+\left|\nabla v_{n}\right|^{2}\right) d x \longrightarrow 0,
\end{aligned}
$$

which contradicts with Lemma 12. If (ii) holds, then we have

$$
\begin{aligned}
m & \geq \limsup _{n \longrightarrow \infty} m_{q_{n}}=\limsup _{n \longrightarrow \infty}\left[I_{q_{n}}\left(u_{n}, v_{n}\right)-\frac{1}{N} J_{q_{n}}\left(u_{n}, v_{n}\right)\right] \\
& =\limsup _{n \longrightarrow \infty}\left[\frac{1}{N} \int_{\mathbb{R}^{N}}\left(\left|\nabla u_{n}\right|^{2}+\left|\nabla v_{n}\right|^{2}\right) d x\right] \\
& \geq \limsup _{n \longrightarrow \infty} \frac{1}{N} \int_{\mathbb{R}^{N}}\left|\nabla v_{n}\right|^{2} d x \geq \frac{1}{N} S^{N / 2} .
\end{aligned}
$$

This is a contradiction. So $u \neq 0$ and through Remark 3, we know that $v \neq 0$. Applying the weak lower-semicontinuity of the norm, we have 


$$
\begin{aligned}
m & \leq I(u, v)=I(u, v)-\frac{1}{N} J(u, v)=\frac{1}{N} \int_{\mathbb{R}^{N}}\left(|\nabla u|^{2}+|\nabla v|^{2}\right) d x \\
& \leq \liminf _{n \longrightarrow \infty} \frac{1}{N} \int_{\mathbb{R}^{N}}\left(\left|\nabla u_{n}\right|^{2}+\left|\nabla v_{n}\right|^{2}\right) d x \\
& =\liminf _{n \longrightarrow \infty}\left[I_{q_{n}}\left(u_{n}, v_{n}\right)-\frac{1}{N} J_{q_{n}}\left(u_{n}, v_{n}\right)\right]=\underset{n \longrightarrow \infty}{\lim \inf _{n \rightarrow \infty} m_{q_{n}} \leq m .}
\end{aligned}
$$

This implies $I(u, v)=m$. We complete the proof.

\section{Data Availability}

The findings in this research do not make use of data.

\section{Conflicts of Interest}

The authors declare that they have no conflicts of interest.

\section{Acknowledgments}

This study was supported by the National Natural Science Foundation of China (11861052), Science and Technology Foundation of Guizhou Province ([2019]1300, [2019]5653), Innovation group project of Department of Education of Guizhou Province ([2019]067), and Foundation of Qiannan Normal University for Nationalities (QNYSKYTD2018012).

\section{References}

[1] N. Akhmediev and A. Ankiewicz, "Novel soliton states and bifurcation phenomena in nonlinear fiber couplers," Physical Review Letters, vol. 70, no. 16, pp. 2395-2398, 1993.

[2] A. Ambrosetti, E. Colorado, and D. Ruiz, "Multi-bump solitons to linearly coupled systems of nonlinear Schrödinger equations," Calculus of Variations and Partial Differential Equations, vol. 30, no. 1, pp. 85-112, 2007.

[3] A. Ambrosetti, G. Cerami, and D. Ruiz, "Solitons of linearly coupled systems of semilinear non-autonomous equations on $\mathbb{R}^{N}$," Journal of Functional Analysis, vol. 254, no. 11, pp. 2816-2845, 2008.

[4] H. D. Liu and Z. L. Liu, "A coupled Schrödinger system with critical exponent," Calculus of Variations and Partial Differential Equations, vol. 59, no. 5, p. 28, 2020.

[5] B. Noris and M. Ramos, "Existence and bounds of positive solutions for a nonlinear Schrödinger system," Proceedings of the American Mathematical Society, vol. 138, no. 5, pp. 1681-1692, 2010.

[6] Z. Chen and W. Zou, "Ground states for a system of Schrodinger equations with critical exponent," Journal of Functional Analysis, vol. 262, no. 7, pp. 3091-3107, 2012.

[7] G. Li and X. H. Tang, "Nehari-type ground state solutions for Schrodinger equations including critical exponent," Applied Mathematics Letters, vol. 37, pp. 101-106, 2014.

[8] A. Bahri and P. L. Lions, "On the existence of a positive solution of semilinear elliptic equations in unbounded domains," Annales de l'Institut Henri Poincaré C, Analyse non linéaire, vol. 14, no. 3, pp. 365-413, 1997.
[9] H. Berestycki and P. L. Lions, "Nonlinear scalar field equations. I existence of a ground state," Archive for Rational Mechanics and Analysis, vol. 82, no. 4, pp. 313-345, 1983.

[10] W. A. Strauss, "Existence of solitary waves in higher dimensions," Communications in Mathematical Physics, vol. 55, no. 2, pp. 149-162, 1977.

[11] W. Xie, H. Chen, and T.-F. Wu, "Ground state solutions for a class of Schrödinger-Poisson systems with Hartree-type nonlinearity," Applicable Analysis, vol. 100, no. 13, pp. 27772803, 2021.

[12] V. S. Guliyev, M. N. Omarova, M. A. Ragusa, and A. Scapellato, "Regularity of solutions of elliptic equations in divergence form in modified local generalized Morrey spaces," Analysis and Mathematical Physics, vol. 11, no. 1, p. 20, 2021.

[13] N. S. Papageorgiou and A. Scapellato, "Multiple solutions for Robin $(p, q)$-equations plus an indefinite potential and a reaction concave near the origin," Analysis and Mathematical Physics, vol. 11, no. 2, p. 27, 2021.

[14] N. S. Papageorgiou and A. Scapellato, "Nonlinear singular problems with convection," Journal of Differential Equations, vol. 296, pp. 493-511, 2021.

[15] C. Keller, "Large-time asymptotic behavior of solutions of nonlinear wave equations perturbed from a stationary ground state," Communications in Partial Differential Equations, vol. 8, no. 10, pp. 1073-1099, 1983.

[16] J. Liu, J.-F. Liao, and C.-L. Tang, “Ground state solution for a class of Schrödinger equations involving general critical growth term," Nonlinearity, vol. 30, no. 3, pp. 899-911, 2017.

[17] M. Willem, "Minimax theorems," in Progress in Nonlinear Differential Equations and their Applications, vol. 24, Birkhäuser Boston, Inc., Boston, MA, USA, 1996.

[18] H. Brezis and E. H. Lieb, "Minimum action solutions of some vector field equations," Communications in Mathematical Physics, vol. 96, no. 1, pp. 97-113, 1984.

[19] Z. J. Chen and W. M. Zou, "On coupled systems of Schrödinger equations," Advances in Differential Equations, vol. 16, pp. 775-800, 2011. 